\title{
D etección indirecta de portadores de fibrosis quística en dos familias chilenas mediante análisis de polimorfismos en el ADN asociados fuertemente al gen CFTR
}

\author{
Nora Riveros $K^{a}$, Juan Ríos $\mathbf{H}^{\mathbf{b}}$. \\ Indirect cystic fibrosis carrier \\ detection in two Chilean families by \\ analysis of DNA polymorphisms \\ closely linked to the CFTR gene
}

Background: Molecular genetic analysis is the only method that allows accurate detection of Cystic Fibrosis (CF) carriers. Nevertheless, its application is restricted to those families in which the affected child holds known mutations. Since most Chilean CF patients already studied are heterozygous and carry a mutation not yet characterized, direct identification of carriers is limited. Linkage analysis of Restriction Fragment Length Polymorphisms (RFLP), using DNA markers closely linked to the CFTR gene, is a useful tool for the detection of carriers in families in which the patient carries an unknown mutation. Aim: To emphasize the usefulness of KM19 and MetH markers for RFLP analysis in extended genealogies in order to identify carriers of unknown mutations. Material and Methods: Selection of three families, in which a sibling of an index case, identified as $\Delta \mathrm{F}-508 /$ unknown mutation, could not be identified as heterozygous carrier or a normal homozygous. Haplotypic characterization for KM19 and MetH markers was performed by PCR amplification of genomic DNA followed by allele specific restriction enzymatic digestion. Results: The siblings of two families were identified as carriers and the sibling of the third family was identified as normal. Conclusions: KM19 and MetH haplotypic analysis provided a rapid method for carrier detection in the families under study. The analysis may be used as a supplement to direct genetic diagnosis and be helpful in genetic counseling (Rev Méd Chile 2005; 133: 648-54).

(Key Words: Cystic fibrosis; Genetic diseases, inborn; Polymorphism, genetic)

Recibido el 12 de marzo, 2004. Aceptado en versión corregida el 30 de marzo, 2005.

Programa de Biología Celular y Molecular. ICBM. Facultad de Medicina de la Universidad de Chile, Santiago de Chile.

aQuímico Farmacéutico, Doctor en Ciencias con mención en Biología.

bioquímico.

Correspondencia a: Dra. Nora Riveros K. Programa de Biología Celular y Molecular. ICBM. Facultad de Medicina, Universidad de Chile. Independencia 1027. Santiago Chile. Fax: 7355580. E mail: nriveros@med.uchile.cl. 
L a fibrosis quística (FQ) es una enfermedad letal, de herencia autosómica recesiva, cuya incidencia en la población chilena se estima en 1 de cada 4.000 nacidos vivos. Considerando que el promedio de sobrevida de los enfermos en nuestro país es de 12 años, se calcula que actualmente debieran existir en Chile aproximadamente 400 niños vivos afectados de FQ.

La medición de electrolitos en el sudor aun cuando constituye una excelente herramienta de diagnóstico que debe aplicarse siempre ante la sospecha de un caso de FQ, no es siempre concluyente. Hay pacientes, con sintomatología altamente sugerente de la enfermedad, que presentan una concentración de cloruro normal o limítrofe, en cuyo caso sólo el análisis genético molecular permite confirmar un diagnóstico clínico dudoso 1,2 .

El gen alterado en la FQ codifica la proteína CFTR, reguladora del transporte epitelial de cloruro. Se han descrito más de 900 mutaciones diferentes a lo largo del gen, al menos un ciento de ellas asociadas a la expresión clínica de la enfermedad. Las mutaciones se presentan con frecuencia variable según el origen étnico de la población, la más común, presente en $70 \%$ de los alelos FQ en pacientes caucásicos, consiste en la deleción de 3 pares de bases y se traduce en la pérdida de una fenilalanina en la posición 508 de la proteína CFTR ( $\Delta \mathrm{F}-508)$. Los enfermos restantes presentan diferentes mutaciones, cuya frecuencia individual no supera al 3\%3,4. De esto se desprende que el diagnóstico molecular de rutina, mediante el análisis de cada una de las mutaciones asociadas a la enfermedad, resulta impracticable sin la identificación previa de las mutaciones más comunes en la población en que se aplica.

El uso de kits comerciales, disponibles en nuestro país, permite el análisis simultáneo de varias mutaciones. Sin embargo, la tasa de detección depende de cuán frecuentes sean estas mutaciones en la población. Es así como el uso del panel recomendado por The American College of Medical Genetics, que comprende las 25 mutaciones más comunes en la población caucásica, permite un nivel de detección promedio inferior a $60 \%$ de los alelos mutados en la población hispanoamericana ${ }^{5}$. Por lo tanto, un resultado negativo en este análisis, no permite descartar la presencia de FQ. Es posible en estos casos, aunque su costo es elevado, recurrir al servicio de empresas que cuentan con laboratorios especializados en el extranjero y que ofrecen el análisis de todas las mutaciones que figuran en The Cystic Fibrosis Mutation Data Base.

En el primer estudio realizado en pacientes chilenos, mediante el análisis de 9 mutaciones, descritas entre las más frecuentes en la población hispana, caracterizamos $40 \%$ de los alelos mutados $^{1}$. Cincuenta por ciento de los 24 pacientes resultaron heterocigotos $\Delta \mathrm{F}-508 /$ mutación no identificada. En un estudio posterior, en el que se analizan 20 mutaciones, se caracterizó a $66 \%$ de los alelos mutados, determinándose que $44 \%$ de los pacientes corresponden a heterocigotos $\Delta \mathrm{F}$ $508 /$ mutación no identificada $(n=25)^{6}$.

La presencia mayoritaria de individuos FQ heterocigotos, en que sólo se ha caracterizado la mutación presente en uno de sus alelos, imposibilita la aplicación del análisis molecular directo para la detección de portadores. Dada la gravedad de esta enfermedad, y el alto costo que representa el tratamiento y cuidado de un niño con FQ, la identificación de portadores a partir del núcleo familiar de individuos afectados, reviste gran importancia.

El estudio de regiones cromosómicas adyacentes al gen CFTR ha permitido identificar varios polimorfismos en el largo de fragmentos de restricción (RFLP), fuertemente ligados a este gen $^{7-9}$. El análisis de la segregación de los alelos de marcadores polimórficos, a través de genealogías extendidas, permite caracterizar haplotipos de esta región cromosómica y la detección indirecta de portadores en aquellas familias en que el enfermo es heterocigoto para una mutación desconocida $^{10-15}$.

En este trabajo se muestra la aplicación de este tipo de análisis en familias seleccionadas a partir de un caso índice afectado con FQ, cuyo genotipo ha sido caracterizado como heterocigoto $\Delta \mathrm{F}-508$ / mutación no identificada. La caracterización haplotípica se realizó mediante el uso de endonucleasas de restricción que cortan en secuencias específicas del ADN y que permiten la discriminación de dos formas alélicas según esté o no presente el sitio de reconocimiento de la enzima. Los marcadores polimórficos analizados fueron MetH y KM19, localizados en los loci MET y D7S23, respectivamente, río arriba del gen CFTR. 
KM19 se localiza $125 \mathrm{~kb}$ río arriba del promotor del gen CFTR y aproximadamente a $225 \mathrm{~kb}$ de $\Delta \mathrm{F}$ 508 y el locus MET se ubica alrededor de $1450 \mathrm{~kb}$ del extremo 3' de CFTR ${ }^{7,9}$.

\section{MATERIAL Y MÉTODO}

En una muestra de familias pesquisadas a partir de casos índices afectados de FQ, incluidas en dos estudios publicados anteriormente ${ }^{1,16}$, se seleccionaron 3 de ellas, en que un hermano del caso índice, caracterizado como $\Delta \mathrm{F}-508 /$ mutación desconocida, no podía ser tipificado como portador u homocigoto normal.

Método. La obtención de ADN genómico y la tipificación de la mutación $\Delta$ F-508 se realizó de acuerdo a lo descrito por Ríos y $\mathrm{col}^{16}$. Para la caracterización de los polimorfismos, se amplificaron regiones de los loci MET y D7S23, que contienen sitios polimórficos para las enzimas MspI (MetH) y PstI (KM19), respectivamente ${ }^{10,17}$ (Figura 1). La amplificación se realizó mediante la técnica de PCR usando los siguientes partidores:
KM19:

5'- ATTCTGTCCAGGAAACTTTGTGTTTTGTCA-3"'

5'- GTCTAAAGGGTATCAGTCCAAAAATGAAT-3'

MetH:

\section{5'- CATCCAATGTAGGAGAGCCTTAGTC-3' \\ 5'- CCATTTTTGTGTCTTCTAGTCTAAGG-3'}

Las condiciones de la PCR fueron: un ciclo inicial de 5 min a $95^{\circ}, 35$ ciclos de $1 \mathrm{~min}$ a $95^{\circ}, 1$ min a $50^{\circ}$ y 1 min a $72^{\circ}$, para terminar con 1 ciclo de $10 \mathrm{~min}$ a $72^{\circ}$. Los fragmentos amplificados se incubaron durante la noche con $2 \mathrm{U}$ de enzima/ $\mu \mathrm{g}$ de ADN. El producto de la digestión se analizó mediante electroforesis en geles de agarosa al $2 \%$ a $90 \mathrm{~V}$ por $2 \mathrm{~h}$. El patrón de migración electroforética de los fragmentos de restricción se visualizó al uv, previa tinción del gel con bromuro de etidio.

\section{RESULTADOs}

Los marcadores KM19 y MetH se expresan de forma mendeliana codominante, lo que permite discriminar, sin duda, el genotipo del individuo en ese locus, el haplotipo de cada cromosoma paren-

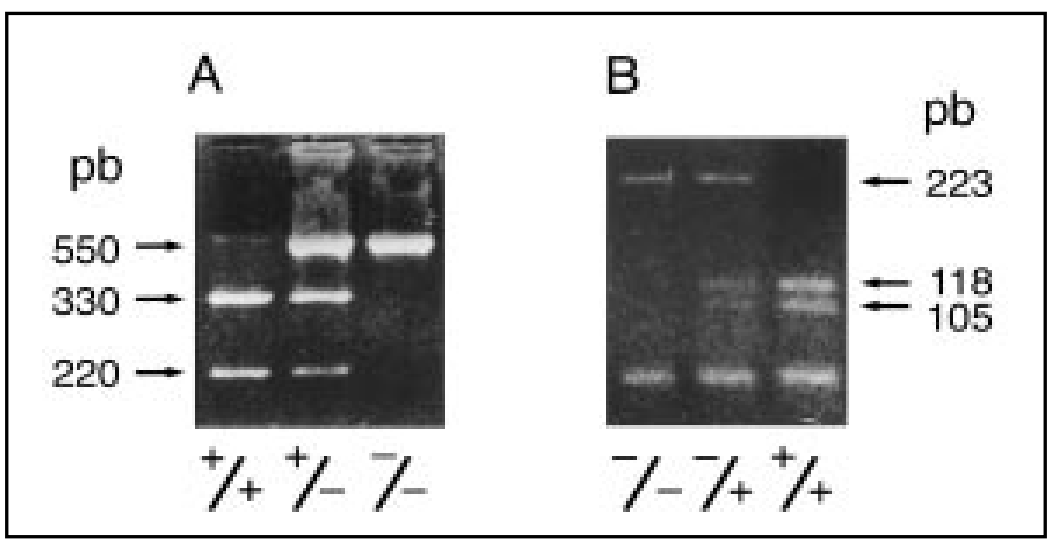

Figura 1. Análisis de polimorfismos mediante PCR y digestión enzimática. Se designa con el signo (+) al alelo que presenta la secuencia reconocida por la enzima de restricción y con (-) al alelo que no la presenta. A. Caracterización para el marcador KM19: se amplificó un fragmento de 550 pb, la digestión del producto amplificado origina fragmentos de 330 y $220 \mathrm{pb}$ en presencia del sitio polimórfico reconocido por la enzima PstI. Carriles: 1 homocigoto $(+/+)$; 2 heterocigoto $(+/-)$; 3 homocigoto $(-/-)$. B. Caracterización para MetH: el producto de amplificación, de $223 \mathrm{pb}$ al ser digerido con MspI origina fragmentos de 118 y $105 \mathrm{pb}$ en presencia del sitio polimórfico, y conserva su tamaño en ausencia de éste. Carriles: 1 homozigoto $(-/-) ; 2$ heterozigoto $(+/-)$; 3 homozigoto $(+/+)$. 
tal se determinó usando el individuo afectado para establecer la fase. Puesto que la FQ es una entidad autosómica recesiva, se establece como axioma que los afectados presentan sus dos alelos CFTR mutados, uno de ellos caracterizado con la deleción $\Delta \mathrm{F}-508$ y el otro portador de una mutación no identificada y que ambos padres son portadores de un alelo mutado. De acuerdo con numerosos antecedentes bibliográficos que así lo indican, se asume además que no ha habido recombinación génica entre los marcadores y el gen $\mathrm{CFTR}^{9-15}$. Ambos marcadores muestran fuerte desequilibrio de ligamiento con el gen CFTR.

Familia 1. El probando es una niña de 7 años, con test del sudor de $86,6 \mathrm{mEq} / \mathrm{L}$ y sintomatología pulmonar. Su padre es portador de la mutación $\Delta \mathrm{F}-508$ y su madre de una mutación no caracterizada. Se desea determinar si su hermano, de 8 años, fenotípicamente normal, que no presenta la mutación $\Delta \mathrm{F}-508$, ha heredado de su madre el alelo normal o el mutado (Figura 2).

Para construir las fases haplotípicas, se parte del haplotipo de la niña afectada, heterozigota para la mutación $\Delta$ F-508 y portadora de una mutación desconocida, quien es $(-/+)$ para el sitio de reconocimiento PstI en la región KM19. Ya que la madre es PstI (-/-), necesariamente la mutación $\Delta \mathrm{F}-508$ se encuentra en fase de acoplamiento con PstI (+) proveniente de su padre, cuyo genotipo es $(-/+)$ en KM19. La determinación del haplotipo en el padre no permite por sí sola establecer la calidad de portador del hermano de la afectada, por cuanto en la madre no puede establecerse la fase entre el alelo mutado sobre CFTR, debido a su condición de homocigoto negativo para PstI. Al tipificar el locus MetH, se determina que el padre es MspI ( $/ /+)$. Como la niña enferma es MspI (-/+), el alelo MspI (-) del padre necesariamente debe estar en fase de acoplamiento con $\Delta$ F-508 y KM19 (+).

La paciente hereda el alelo MetH (-) de su padre y MetH (+) de su madre, en quien este haplotipo se encuentra en fase de acoplamiento con KM19 (-) y una mutación desconocida en el gen CFTR. El hermano de la afectada es $(+/+)$ para MetH y (-/-) para KM19 y no es portador $\Delta \mathrm{F}-508$, por lo tanto, ha heredado de su padre el alelo MetH (+), asociado con el gen CFTR normal y de

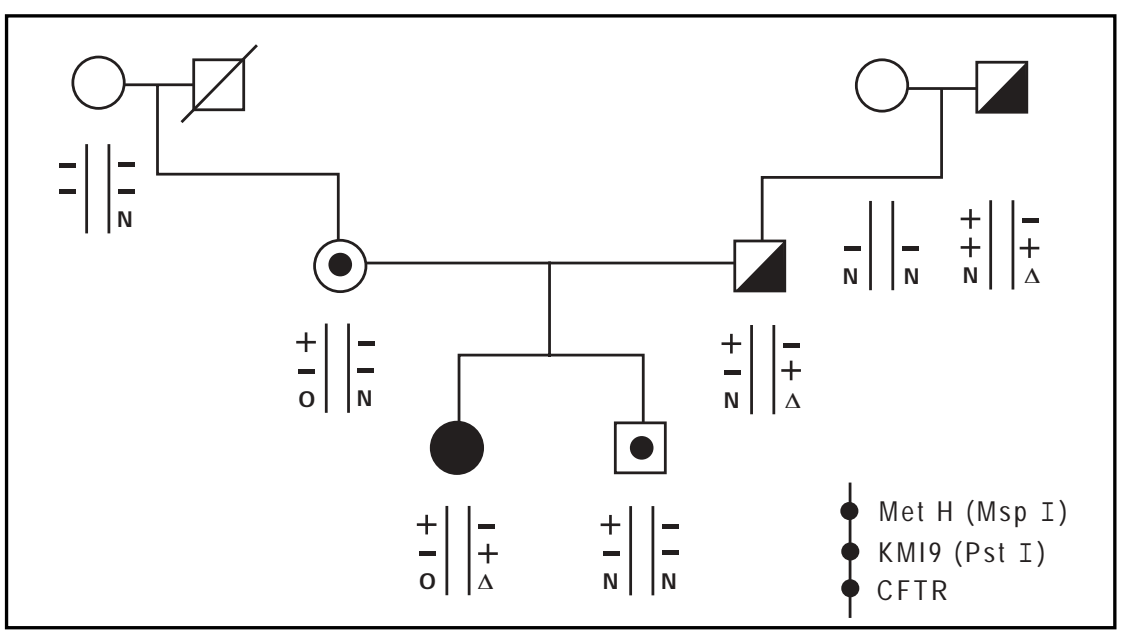

Figura 2. Familia 1: Análisis de polimorfismos para los marcadores MetH y KM19, mediante PCR y digestión enzimática, en una familia cuya hija, caso índice, ha sido caracterizada como heterozigoto $\Delta \mathrm{F}-508 /$ mutación desconocida. Las fases haplotípicas del hermano de la afectada, permiten diagnosticarle como portador de una mutación desconocida en CFTR. En la genealogía los símbolos llenos denotan al individuo afectado, los símbolos con inserto corresponden a portadores y los símbolos abiertos a no portadores. En el ángulo inferior derecho se indica la posición de los marcadores polimórficos con relación a CFTR. Las fases haplotípicas de los marcadores se indican para cada individuo: $(+)$ alelo reconocido por enzima de restricción; $(-)$ alelo que no presenta el sitio polimórfico; $(\Delta) \Delta \mathrm{F}-508 ;(0)$ mutación desconocida; $(\mathrm{N})$ alelo normal. 
su madre el MetH $(+)$ en fase con una mutación desconocida. De este modo las fases haplotípicas del hermano de la afectada, permiten diagnosticarle como portador de una mutación en el gen CFTR, heredada de su madre, sin necesidad de identificar previamente de cuál mutación se trata.

Familia 2. El probando es un niño de 6 años con test del sudor de $116 \mathrm{mEq} / \mathrm{L}$, con sintomatología pulmonar y digestiva. Su madre y su abuela materna son portadoras de la mutación $\Delta \mathrm{F}-508 \mathrm{y}$ su padre de una mutación no identificada Se desea determinar si la hermana de 13 años, fenotípicamente normal, ha heredado de su padre el alelo mutado (Figura 3).

El niño afectado, heterocigoto para la mutación $\Delta$ F-508 y una mutación no determinada, es PstI (-/+). Puesto que la madre es PstI $(t+)$ y portadora de $\Delta \mathrm{F}$ 508 , esta mutación debe estar en fase de acoplamiento con PstI (+). El padre, PstI (-/+), es portador de una mutación no identificada, la que de acuerdo al haplotipo del niño debe estar en fase de acoplamiento con PstI (-). La hermana que es $(-/+)$ para PstI y que no es portadora $\Delta \mathrm{F}-508$ hereda el alelo PstI $(+)$ asociado al gen normal de su madre y el PstI (-), asociado a la mutación desconocida, de su padre. El análisis practicado permite caracterizarla sin ambigüedad como portadora de FQ. Puede establecerse que el alelo Pst $(+)$ asociado a la mutación $\Delta$ F-508 proviene de su abuela matema. La tipificación para el marcador MetH comobora el resultado obtenido para KM19.

Familia 3. El probando es un niño de 6 años con test del sudor de $102 \mathrm{mEq} / \mathrm{L}$, y sintomatología pulmonar. Su padre y su abuela patema son portadores $\Delta \mathrm{F}-508$. La madre es portadora de una mutación no identificada. Se desea determinar la condición de la hermana, de 11 años, fenotípicamente normal, que no presenta la mutación $\Delta \mathrm{F}-508$. (Figura 4). El haplotipo del niño afectado, heterozigoto para la mutación $\Delta$ F-508 y portador de una mutación desconocida es $(t+)$ para Pstl. Ya que el padre es PstI $(-/+)$, la mutación $\Delta \mathrm{F}-508$ se encuentra necesariamente en fase de acoplamiento con PstI (+). Su madre es PstI $(-/+)$, por lo que la mutación desconocida está en fase de acoplamiento con Pst (+). Por lo tanto, la niña, quien presenta Pst (-/-), posee ambos alelos normales. La caracterización de los RFLP para el marcador MetH, confirma su calidad de no portadora.

\section{DisCUSIÓN}

El análisis de haplotipos en genealogías extendidas, basado en los marcadores MetH y KM19 ligados estrechamente con el gen CFTR, practica-

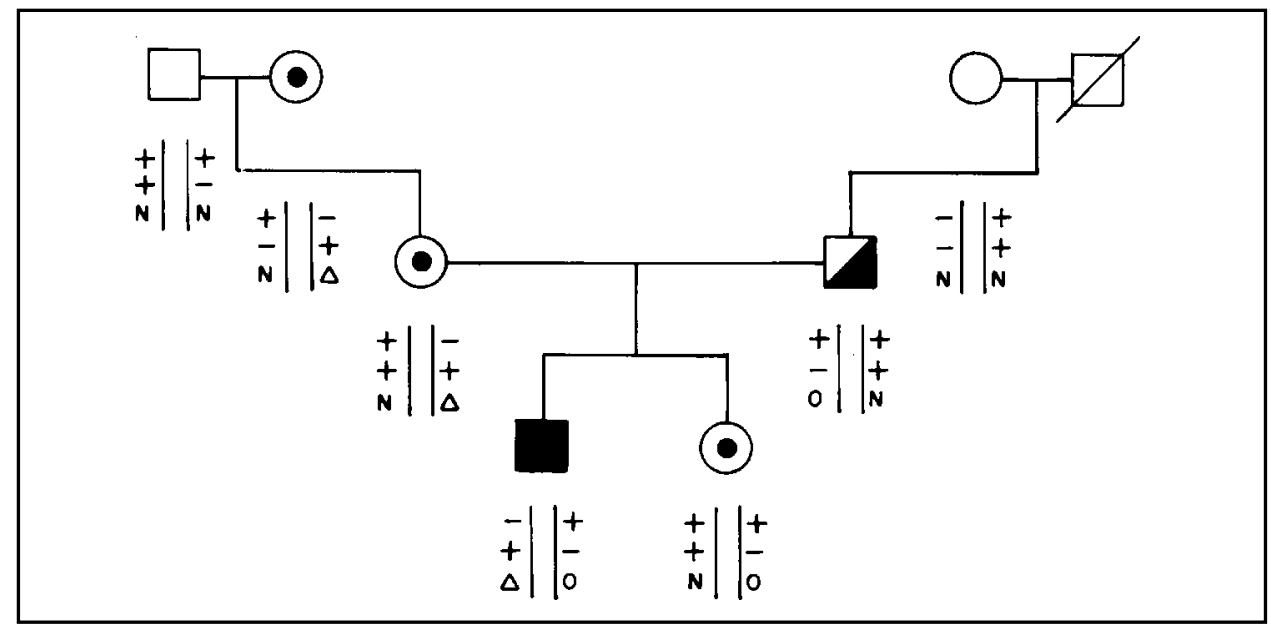

Figura 3. Familia 2: Análisis de polimorfismos para los mancadores MetH y KM19, en una familia cuyo hijo, caso índice, ha sido caracterizado como heterozigoto $\Delta$ F-508/mutación desconocida. Las fases haplotípicas de su hermana, permiten establecer su carácter de portadora de una mutación desconocida. Los símbolos y el método usado se indican en la Figura 2. 


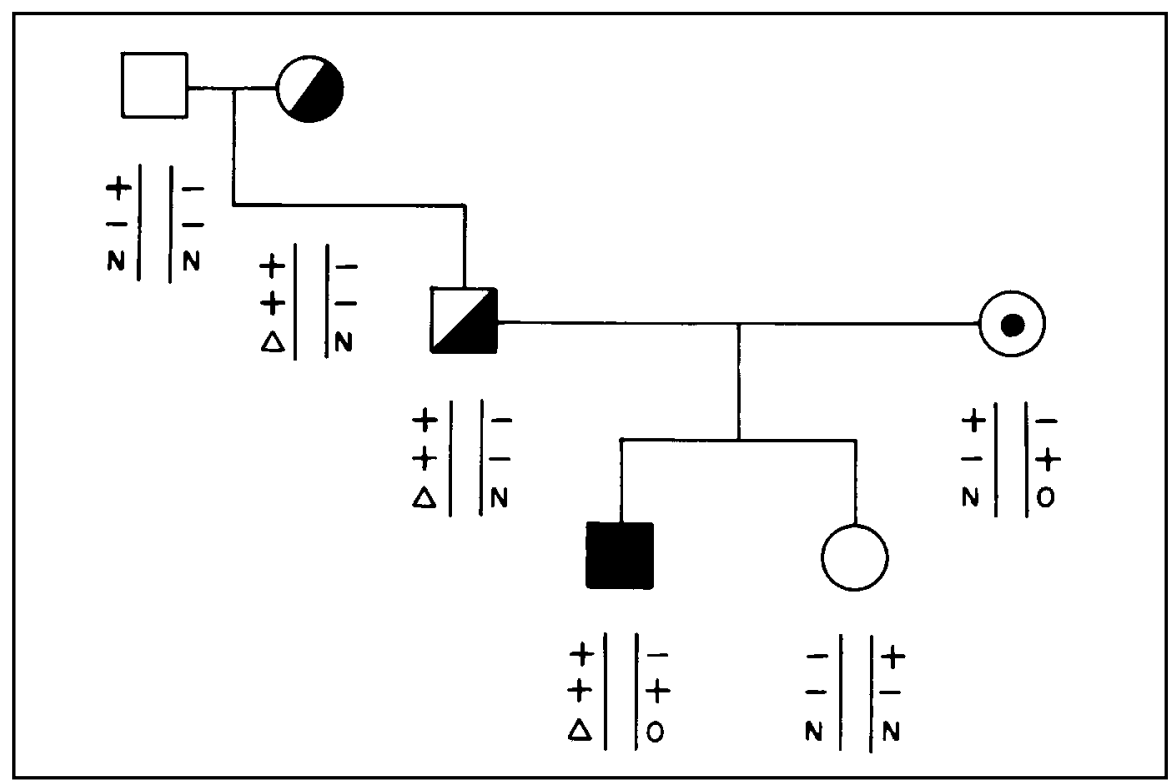

Figura 4. Familia 3: Análisis de polimorfismos para los marcadores MetH y KM19, en una familia cuyo hijo, caso índice, ha sido caracterizado como heterocigoto $\Delta$ F-508/mutación desconocida. Las fases haplotípicas de su hermana establecen su calidad de no portadora. La simbología y el método usado corresponden a los de la Figura 2.

do a 3 familias, en que el enfermo es heterocigoto para una mutación no caracterizada, permitió establecer en sus hermanos, fenotípicamente sanos, el carácter de portadores (familias 1 y 2) 0 individuos normales (familia 3). La inferencia de la calidad de portador, de acuerdo al haplotipo determinado, está dada por la baja fracción de recombinación para ambos marcadores, ampliamente documentada en la literatura ${ }^{7-18}$.

Dado el alto porcentaje de heterocigotos afectados por una mutación desconocida, entre los pacientes chilenos ${ }^{1,6}$, debido al gran número de mutaciones descritas en el gen CFTR y al alto costo que significaría el análisis de cada una de ellas, la aplicación de este tipo de análisis constituye una buena alternativa para la detección de portadores.

$\mathrm{Si}$ bien el uso de un número limitado de marcadores no siempre permite establecer la fase haplotípica para la mutación dentro de una familia, la inclusión de marcadores intragénicos, como microsatélites localizados en regiones intrónicas u otros más cercanos a la mutación causal, disminuyen la posibilidad de recombinación entre ambas regiones y el riesgo de falsos positivos o falsos negativos ${ }^{19,20}$.
El análisis de polimorfismos se ha usado extensamente en diagnóstico prenatal, lo que reviste enorme importancia en familias de alto riesgo, ya que posibilita el inicio de un tratamiento precoz de la enfermedad, contribuyendo a un mejor pronóstico y calidad de vida para el recién nacido ${ }^{5}$. Por otra parte, la asesoría genética que pueda brindarse a parejas de individuos portadores, permite a ellos asumir una paternidad responsable y estar atentos a la aparición de síntomas sugerentes de la enfermedad en sus hijos. Esta técnica también puede ser utilizada para el diagnóstico de individuos con antecedentes familiares y sintomatología sugerente de FQ, aun cuando se desconozcan las mutaciones específicas que afectan a ambos alelos en el integrante de la familia diagnosticado con la enfermedad.

Este trabajo constituye un ejemplo particular de una técnica de diagnóstico indirecto de gran potencialidad, aplicable a cualquier patología originada por mutaciones genéticas si se conoce la posición del gen a nivel cromosomal y se cuenta con marcadores moleculares colindantes con los que segregue en desequilibrio de ligamiento. 


\section{REFERENCIAS}

1. Ríos J, Oremana O, Aspilaga M, Avendaño L, Largo I, Riveros N. CFTR mutations in Chilean cystic fibrosis patients. Hum Genet 1994; 94: 291-4.

2. Sánchez I, Pérez MA, Boza ML, LezanaV, Vila A, Repetto G et al. Consenso nacional de fibrosis quística. Rev Chil Pediatr 2001; 72: 356-80.

3. Zielinski J, Tsui L Cystic Fibrosis: Genotypic and Phenotypic variations. Ann Rev Genetics 1995; 29: 777-87.

4. Cystic Fibrosis Genetic Analysis Consortium. CFTR mutation data 2000. http//www.genet. sickkids.on.ca./ cftr/.

5. Tait J, Gibson R, Marshall S, Cheng E, Stern D, Cutting G. Cystic Fibrosis. Gene Clinics 2001; 119.

6. Repetto G, Poggi H, Harris P, Navarro H, Sánchez I, GuiRALDES E ET AL. Identificación de mutaciones en el gen CFTR en pacientes chilenos con fibrosis quística. Rev Méd Chile 2001; 129: 841-7.

7. Kerem B, Rommens J, Buchanan J, Markiewicz D, Cox T, Chakravarti A et al. Identification of the Cystic Fibrosis Gene: Genetic Analysis. Science 1989; 245: 1073-9.

8. Lathrop GM, Farrall M, O'Conell P, Wainwright B, LEPPERT M, NAKAMURA Y ET AL. Refined linkage map of Chromosome 7 in the region of the cystic fibrosis gene. Am J Hum Genet 1988; 42: 38-44.

9. Cutting G, Antonarakis S, Buetow K, Kasch L, Rosenstein B, Kazazian H. Analysis of DNA polymorphism haplotypes linked to the Cystic Fibrosis locus in North American black and Caucasian families supports the existence of multiple mutations of the cystic fibrosis gene. Am J Hum Genet 1989; 44: 307-18.

10. Estivill $X$, Scambler PJ, Wainwright BJ, Hawley $K$, Frederick P, Schartz M et al. Patterns of polymorphisms and linkage disequilibrium for cystic fibrosis. Genomics 1987; 1: 257-63.

11. Anwar R, Murray K, Hedge PJ, Smith JC, Markham AF. DNA sequence analysis of the KM19 locus linked to cystic fibrosis. Design of new oligonucleotides to remove non specific PCR products. Hum Genetics 1990; 85: 319-23.

12. SyväNen AM, Sajantila A, LuKKa M. Identification of individuals by analysis of biallelic DNA markers using PCR and solid phase minisequencing. Am J Hum Genet 1993; 52: 46-59.

13. Farrall M, Watson E, Bates G, Bell J, Davies KA, EstrviL $\mathrm{X}$ et aL. Further data supporting linkage between $\mathrm{CF}$ and the met oncogene and haplotype analysis with met and pJ3.11. Am J Hum Genet 1986; 30: 713-9.

14. Watkins PC, Shwartz R, Hoffman N, Doherty R, KUINGER KW. A linkage study of cystic fibrosis in extended multigenerational pedigrees. Am J Hum Genet 1986; 39: 35-43.

15. Dean M, O’Connell P, Leppert M, Park M, Amos JA, White B ET AL. Three additional DNA polymorphisms in the met gene and D7S8 locus: use in prenatal diagnosis of cystic fibrosis. J Pediatr 1987; 111: 490-5.

16. Ríos J, Oreinana O, Riveros N. Análisis genético molecular de la fibrosis quística en dos pacientes chilenos y sus familias. Rev Méd Chile 1994; 122: 13-8.

17. Horn G, Richards B, Merrill JJ, Kunger KW. Characterization and rapid diagnostic analysis of DNA polymorphisms closely linked to the cystic fibrosis locus. Clin Chem 1990; 36: 1614-9.

18. Beaudet A, Feldman S, Ferndach G, Buffone G, OBRIAN WE. Linkage disequilibrium, cystic fibrosis and genetic counseling. Am J Hum Genet 1989; 44: 319-26.

19. Magnani C, Cremonesi L, Be山loni E, Ferrari M, Seia M, Devoto M ET AL. Informativity of intragenic microsatellites for carrier detection and prenatal diagnosis of cystic fibrosis in the italian population. Clin Genet 1994; 45: 135-9.

20. Morral N, Nunes V, Casals T, Chiшón M, Giménez J, BERTRANPETIT J ET AL. Microsatellite haplotypes for cystic fibrosis: mutation frameworks and evolutionary tracers. Human Molecular Genetics 1993; 2: $1015-22$

Agradecimientos:

Agradecemos al Dr. Mauricio Arcos Burgos, Médico Cirujano, PhD Ciencias Biomédicas, por su contribución en la obtención e interpretación de resultados. 